\title{
LETTER
}

\section{The long-term metabolic outcomes of dysglycemia during critical illness}

\author{
Lavi Oud* \\ See related research by Gornik et al., http://ccforum.com/content/14/4/R130
}

Gornik and colleagues should be commended for providing the first report on the long-term glucose metabolism sequelae in survivors of critical illness with no apparent dysglycemia shortly after hospital discharge [1]. The authors hypothesized that hyperglycemia in the intensive care unit unmasks predisposition for future development of diabetes. The interpretation of their findings, however, is limited by several aspects of their methodology.

No systematic control for covariates and confounders was performed. Their cohort was divided, as acknowledged by the authors, by an arbitrary (for the purpose of long-term prediction) glycemic cutoff value. While intuitively appealing, the latter is not necessarily the optimal predictor of future risk of impaired glucose metabolism. When considering the whole cohort of patients with a normal first postdischarge oral glucose tolerance test (the eligible cohort), what was the predictive role for traditional predictors of diabetes mellitus and prediabetes mellitus (DM/pre-DM) (for example, age, family history, body mass index) and for inhospital variables (for example, severity of illness)? It would also be instructive to explore the role of previously studied glycemic indices - such as admission, maximal and mean glucose values - to determine the best predictive cutoff value for future development of DM/pre-DM.

In addition, almost $40 \%$ of the eligible cohort was excluded from analysis due to death or discontinuation of follow-up. How many of these patients who had at least the first 12-month follow-up were diagnosed with DM/ pre-DM?

Because DM/pre-DM are commonly under-recognized [2], these analyses can provide initial predictive modeling, which may inform future development of public health policy for earlier detection and intervention in survivors of critical illness.

\section{Authors' response}

Ivan Gornik

We appreciate Dr Oud's sound comments to the limitations of our study [1]; we will try to address them all.

Analysis of covariates was performed, although the results were not included in the paper. Family history of diabetes and body mass index were, in univariate analyses, associated with higher 5-year incidence of diabetes; higher Acute Physiology and Chronic Health Evaluation II score was associated with lower 5-year incidence of diabetes. A logistic regression model that included age, family history of diabetes, body mass index, Acute Physiology and Chronic Health Evaluation II score and intensive care unit

${ }^{*}$ Correspondence: lavi.oud@ttuhsc.edu

Division of Pulmonary and Critical Care Medicine, Department of Internal Medicine, Texas Tech University Health Sciences Center at the Permian Basin, 701 West $5^{\text {th }}$ Street, Odessa, TX 79763, USA hyperglycemia revealed only the final variable as an independent predictor of future diabetes (Table 1).

Severity of illness was associated with lower risk of diabetes. We have also previously reported the incidence of diabetes following hyperglycemia in sepsis according to the severity of disease [3]. Relative risks for DM in patients who had hyperglycemia in mild sepsis and septic shock were 6.94 and 2.20 , respectively. The hypothesis is that more severe illness can produce hyperglycemia more frequently even in patients without a predisposition to diabetes, therefore reducing the discriminating capability.

Besides the chosen threshold of $7.7 \mathrm{mmol} / \mathrm{l}$, other possible thresholds and glycemic variables (admission, highest and mean glucose) were possible predictors of future diabetes, but none showed better results in our population. Future studies may show their value, especially in populations other than medical intensive care unit patients. 
Table 1. Logistic regression model for 5-year risk of diabetes mellitus in patients admitted to the ICU

\begin{tabular}{lcc}
\hline Independent variable & Odds ratio $(\mathbf{9 5 \%} \mathrm{Cl})$ & $P$ value \\
\hline Hyperglycemia $(>7.7 \mathrm{mmol} / \mathrm{l})$ in the ICU & $5.04(2.48$ to 10.26$)$ & $<0.001$ \\
Body mass index $\left(\mathrm{kg} / \mathrm{m}^{2}\right)$ - for each increment of $1 \mathrm{~kg} / \mathrm{m}^{2}$ & $1.44(0.93$ to 2.22$)$ & 0.10 \\
Age (years) - for each increment of 1 year & $0.89(0.77$ to 1.03$)$ & 0.13 \\
Family history of diabetes & $0.87(0.22$ to 2.03$)$ & 0.48 \\
APACHE II score - for each increment of 1 point & $0.63(0.13$ to 1.19) & 0.09 \\
\hline
\end{tabular}

APACHE, Acute Physiology and Chronic Health Evaluation; $\mathrm{Cl}$, confidence interval; ICU, intensive care unit.

The dropout rate was indeed high and the numbers of patients decreased after the first years of follow-up. The difference in the incidence of diabetes became significant only after 2 years: $3.1 \%$ of patients $(9 / 290)$ in the hyperglycemia group and $0.7 \%$ of patients $(4 / 539)$ in the normoglycemia group were diagnosed with diabetes $(P=0.023)$. The 2-year period could therefore be the minimal follow-up target in future studies.

Abbreviations

DM, diabetes mellitus.

\section{Competing interests}

The author declares that he has no competing interests.
Published: 2 December 2010

\section{References}

1. Gornik I, Vujaklija-Brajković A, Pavlić Renar I, Gašparović V: A prospective observational study of the relationship of critical illness associated hyperglycaemia in medical ICU patients and subsequent development of type 2 diabetes. Crit Care 2010, 14:R130.

2. National Diabetes Statistics 2007 [http://diabetes.niddk.nih.gov/dm/pubs/ statistics/index.htm]

3. Gornik I, Vujaklija A, Lukic E, Madzarac G, Gasparovic V: Hyperglycemia in sepsis is a risk factor for development of type II diabetes. J Crit Care 2009, 25:263-269.

doi:10.1186/cc9321

Cite this article as: Oud L: The long-term metabolic outcomes of dysglycemia during critical illness. Critical Care 2010, 14:452. 